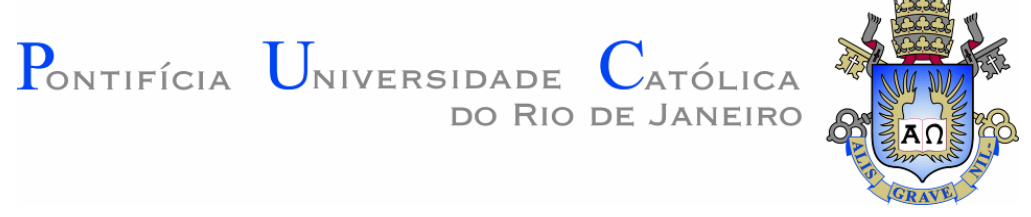

Kelly Margarita Colmenares Vargas

Processo de Deslocamento de Óleo em Micro Modelos de Meios Porosos por Injeção de Emulsão de Óleo em Água

Dissertação de Mestrado

Dissertação apresentada como requisito parcial para obtenção do título de Mestre pelo Programa de Pós Graduação em Engenharia Mecânica da PUC - Rio.

Prof. Márcio da Silveira Carvalho

Orientador

Departamento de Engenharia Mecânica - PUC-Rio

Rio de Janeiro

Maio de 2014 


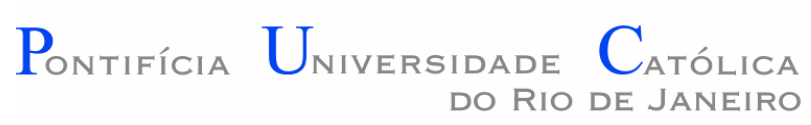

DO RIO DE JANEIRO

Kelly Margarita Colmenares Vargas

\section{Processo de Deslocamento de Óleo em Micro Modelos de Meios Porosos por Injeção de Emulsão de Óleo em Água}

Dissertação apresentada como requisito parcial para obtenção do título de Mestre pelo Programa de Pós Graduação em Engenharia Mecânica da PUC-Rio. Aprovada pela Comissão Examinadora abaixo assinada.

Prof. Márcio da Silveira Carvalho

Orientador

Departamento de Engenharia Mecânica - PUC-Rio

Prof. Paulo Roberto de Sousa Mendes

Departamento de Engenharia Mecânica - PUC-Rio

Prof. Carolina Palma Naveira Cotta Departamento de Engenharia Mecânica - UFRJ

Prof. Mônica Feijó Naccache Departamento de Engenharia Mecânica - PUC-Rio

Prof. José Eugenio Leal Coordenador do Centro Técnico Científico - PUC-Rio

Rio de Janeiro, 12 de Maio de 2014. 
Todos os direitos reservados. É proibida a reprodução total ou parcial do trabalho sem autorização da universidade, do autor e do orientador.

\section{Kelly Margarita Colmenares Vargas}

Gradou-se em Engenharia de Petróleo na Universidad Industrial de Santander - UIS (Santander-Colômbia) em 2008. Anteriormente, atuou como Engenharia de Reservatórios em projetos de pesquisa relacionados com a aplicação de processos de recuperação avançada no Instituto Colombiano do Petróleo.

Ficha Catalográfica

Colmenares Vargas, Kelly Margarita.

Processo de deslocamento de óleo em micro modelos de meios porosos por injeção de emulsão de óleo em água / Kelly Margarita Colmenares Vargas; Orientador: Márcio da Silveira Carvalho. - 2014

$$
146 \text { p.: il. (color.); } 30 \mathrm{~cm}
$$

Dissertação (Mestrado)-Pontifícia Universidade Católica do Rio de Janeiro, Departamento de Engenharia Mecânica, 2012.

Inclui bibliografia

1. Engenharia mecânica - Teses. 2. Recuperação avançada de petróleo. 3. Meios Porosos. 4. Escoamento de Emulsões. 5. Visualização. 6. Micromodelo. I. Carvalho, Márcio da Silveira. II. Pontifícia Universidade Católica do Rio de Janeiro. Departamento de Engenharia Mecânica. III. Título. 
“Talvez não tenha conseguido fazer o melhor, mas lutei para que o melhor fosse feito. Não sou o que deveria ser, mas Graças a Deus, não sou o que era antes". (Martin Luther King) 


\section{Agradecimentos}

A Deus por tudo;

Minha eterna gratidão a minha família, especialmente a minha mãe e aos meus irmãos pelo seu incondicional amor e suporte desde sempre.

Quero agradecer imensamente ao Johann, por tudo o que você é, por partilhar cada instante e por ter estado ao meu lado em todos os momentos durante esta fase importante da minha vida.

Ao meu orientador, Professor Márcio da Silveira Carvalho, pela confiança, e direcionamento, sempre me ajudando e me estimulando durante a realização deste trabalho.

Às pessoas que fizeram parte desses momentos sempre me ajudando e incentivando, e em especial a todos os professores que contribuíram à minha formação.

Ao Departamento de Engenharia Mecânica da Pontifícia Universidade Católica do Rio de Janeiro.

Finalmente, minha gratidão à agência de fomento CAPES pelo apoio financeiro e à PUC-Rio pela oportunidade de estudar em esta instituição, sem os quais este trabalho não poderia ter sido possível. 


\section{Resumo}

Colmenares Vargas, Kelly Margarita; Carvalho, Márcio da Silveira. Processo de Deslocamento de Óleo em Micro Modelos de meios porosos por injeção de emulsão de óleo em água. Rio de Janeiro, 2014. 146p. Dissertação de Mestrado - Departamento de Engenharia Mecânica, Pontifícia Universidade Católica do Rio de Janeiro.

O processo de recuperação de óleo pelo deslocamento com água é o método mais utilizado na indústria de petróleo. No entanto, as altas razões de mobilidade e baixas eficiências de varrido tornam o processo menos eficiente. Uma alternativa usada para minimizar este efeito é a aplicação de tecnologias que atuam como agentes de controle de mobilidade. Dentre eles, e em particular a injeção de emulsões de óleo em água tem sido estudada com relativo sucesso como um método de recuperação avançada de óleo. Alguns estudos indicam melhor varredura do reservatório devido a uma redução da mobilidade da água em regiões do reservatório já varridas por água, mediante a aglomeração e bloqueio parcial dos poros mais permeáveis com gotas da fase dispersa da emulsão. Contudo, ainda não há compreensão plena dos mecanismos associados ao escoamento de emulsões em meios porosos, assim, uma análise e visualização na escala microscópica dos fenômenos envolvidos se faz essencial para a melhora do entendimento do escoamento de emulsões em um reservatório. Neste trabalho, experimentos de escoamento de emulsões foram conduzidos em um micromodelo de vidro, estrutura artificial que busca representar alguns aspectos principais de um meio poroso e proporciona uma adequada visualização do comportamento das faces ao longo do escoamento. Nos experimentos foram realizadas alterações na molhabilidade e variou-se a vazão volumétrica a fim de avaliar diferentes números de capilaridade no meio poroso. Dentro dos resultados mais significativos, foi evidenciado como a fase dispersa da emulsão é capaz de bloquear os poros e gargantas de poro alterando a distribuição dos fluidos no meio poroso, melhorando a eficiência de deslocamento na escala de poro e com isso o fator de recuperação final. Os resultados mostram que, a altos números de capilaridade as forças interfaciais são menos importantes ao reduzir o efeito de bloqueio pelas gotas da fase dispersa nos poros do micromodelo. Estes resultados fornecem um grande aprendizado ao permitir conhecer características do escoamento de emulsões no meio poroso para uma futura aplicação no campo. 


\section{Palavras-chave}

Recuperação avançada de petróleo; meios porosos; escoamento de emulsões; visualização; micromodelo. 


\section{Abstract}

Colmenares Vargas, Kelly Margarita; Carvalho, Márcio da Silveira (Advisor). Oil displacement in micro models of porous media by injection of oil in water emulsion. Rio de Janeiro, 2014. 146p. MSc. Dissertation - Departamento de Engenharia Mecânica, Pontifícia Universidade Católica do Rio de Janeiro.

The oil recovery process by water-flooding is the most used method in the oil industry. However, the high mobility ratios and low sweep efficiencies make the process less effective. A common alternative to minimize this effect is the application of technologies that act as mobility control agents. Among them and in particular the injection of oil in water emulsions has been studied with relative success as an Enhanced Oil Recovery (EOR) method. Several studies indicate a better reservoir sweep due to the water mobility reduction in regions already swept by water. This reduction can be associated with partial blockage of porous media throats by droplets of emulsion dispersed phase. Nevertheless, there is still no full understanding of the mechanisms associated to the flow of emulsions in porous media, thus, an analysis and visualization at the microscopic scale of the involved phenomena is essential for the improvement of the comprehension of the flow of emulsions in a reservoir. In this work, experimental tests related to the flow of emulsions in a glass micro-model were performed, artificial device that represents some principal features of a porous medium and provides a proper visualization of the phase behavior. In the experiments, the effect of the capillary number on the oil recovery factor and the relative influence of the wettability of the porous medium on the oil displacement process were studied. The results evidence how the oil droplets in the emulsion are capable of block the pores and the pore throats modifying the fluids distribution in the porous medium, improving the displacement efficiency at pore scale and consequently the final oil recovery factor. It was also observed that at high capillary numbers, the blocking caused by the capillary pressure needed to deform the droplet becomes less intense. These results provide a great learning by allowing to know the characteristics of the flow of emulsions in porous media for a future field application. 


\section{Keywords}

Enhanced Oil Recovery; porous media; emulsions flow; visualization; micromodel. 


\section{Sumário.}

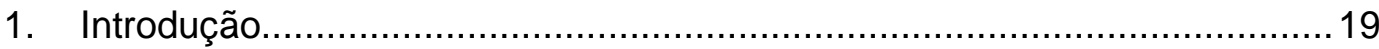

1.1 Escoamento de Emulsões em Meios Porosos ........................................21

1.1.1 Escoamento de Emulsões em Capilares …......................................2 24

1.1.2 Escoamento de Emulsões em Micromodelos .....................................25

1.2 Objetivos e Escopo da Dissertação .....................................................27

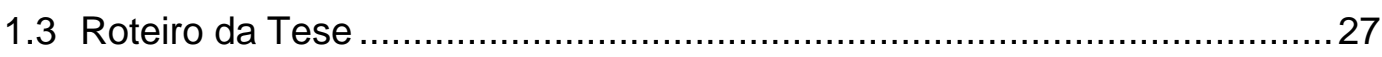

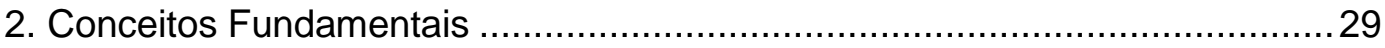

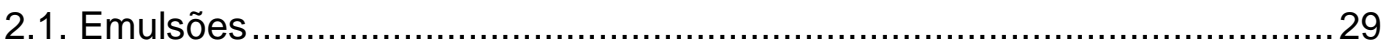

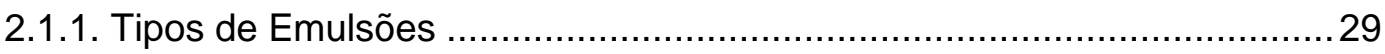

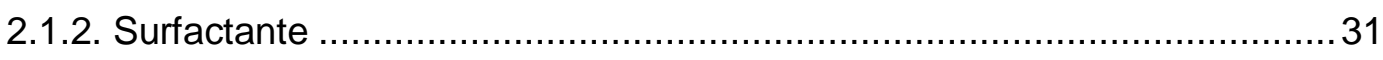

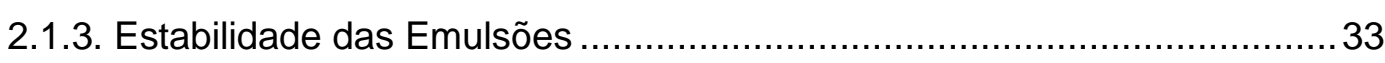

2.2. Micromodelos e sua Utilização em Estudos de Escoamento Bifásico ........37

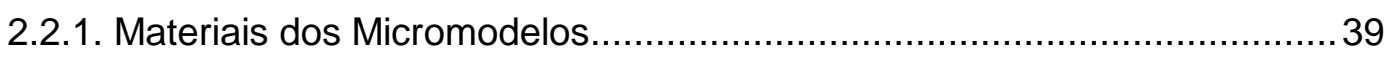

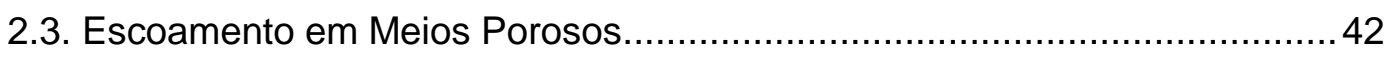

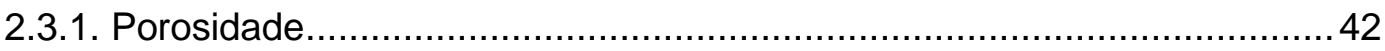

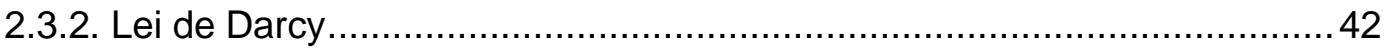

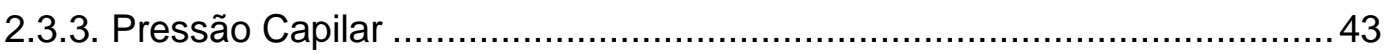

2.3.4. Número de Capilaridade …............................................................... 46

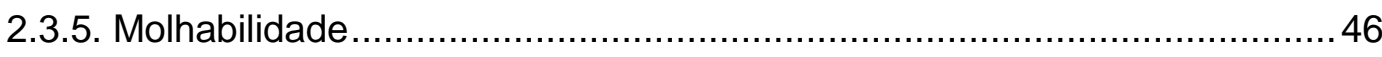

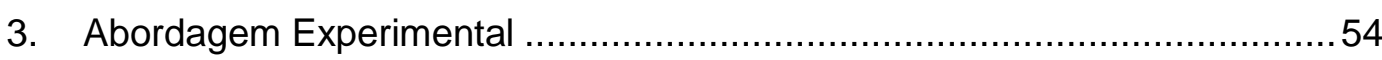

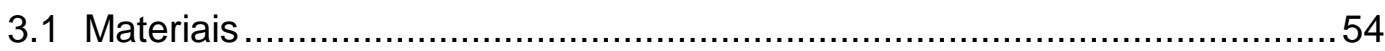

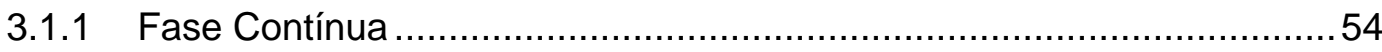

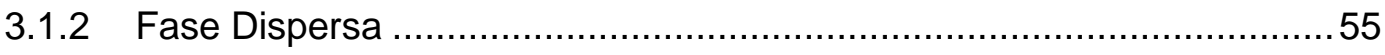

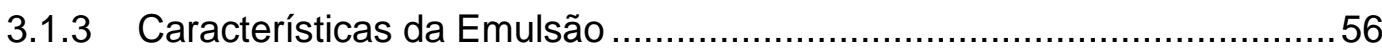

3.1.4 Aditivos Utilizados no Processo de Alteração da Molhabilidade no

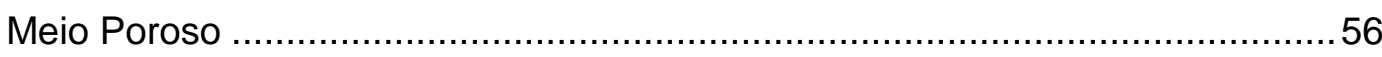

3.2 Preparação e Caracterização das Emulsões ..........................................5 57

3.2.1 Preparação e Técnica de Emulsificação .............................................57

3.2.2 Caracterização das Emulsões .......................................................61

3.3 Determinação da Distribuição de Tamanho de Gota das Emulsões ...........61

3.4 Determinação das Propriedades Interfaciais............................................64 


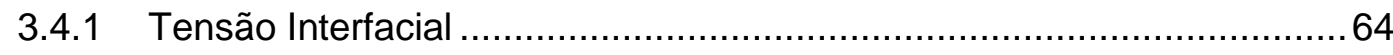

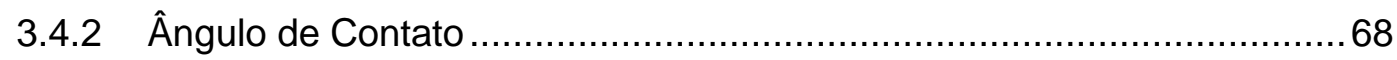

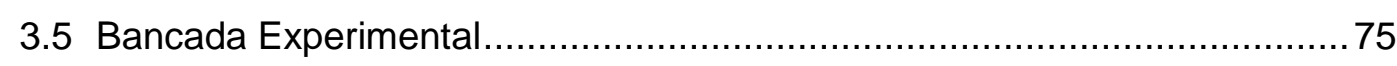

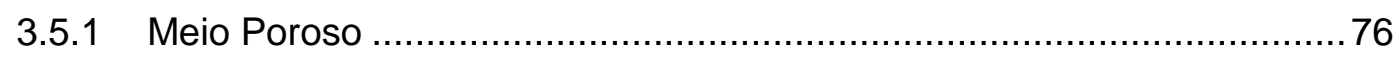

3.5.2 Sistema de Injeção de Fluidos ....................................................... 80

3.5.3 Sistema de Medição da Queda de Pressão ........................................ 81

3.5.4 Sistema de Visualização do Escoamento .......................................... 82

3.6 Descrição do Procedimento Experimental para Visualização do

Deslocamento de Óleo por Injeção de Emulsões O/A ...................................... 85

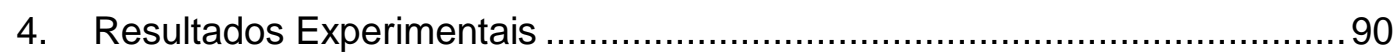

4.1 Saturação do Micromodelo com Água Destilada.....................................90

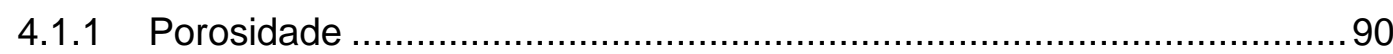

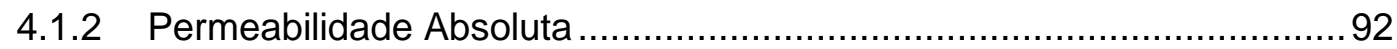

4.2 Visualização do Escoamento de Emulsões através do Micromodelo ...........93

4.2.1 Comportamento do fator de recuperação do óleo e pressão de injeção, em função do número de volumes porosos injetados ...........................94

4.2.2 Efeito do número de capilaridade no processo de deslocamento de óleo por injeção de emulsões no meio poroso ............................................... 108

4.3 Efeito da Molhabilidade no Processo de Deslocamento de Óleo ............... 134

5. Conclusões e Considerações Finais ................................................... 140

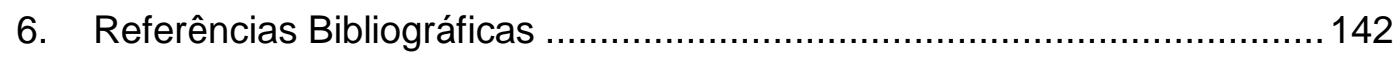




\section{Lista de Figuras.}

Figura 1. 1 Aglomeração e bloqueio de poros com gotículas da emulsão..........20

Figura 1. 2 Representação do fenômeno de Bloqueio. (McAuliffe, 1973) 21

Figura 1. 3 Mecanismos da injeção de emulsões no meio poroso. (Soo and

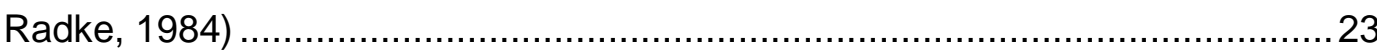

Figura 1. 4 Seção interna do capilar com garganta. (Cobos, 2007)....................24

Figura 1. 5 Sequência de imagens no escoamento de emulsão através do capilar com constrição. (Cobos, 2007)

Figura 1. 6 (a)Captura de Gotas (b) Acumulação de Gotículas e (c)

Coalescência de gotas no meio poroso. (Zeidani and Polikar, 2008)

Figura 1. 7 Processo de aglomeração das gotas de emulsão O/A, em um meio poroso de vidro.

Figura 1. 8 Visualização da mudança do caminho preferencial dos fluidos deslocantes mediante o bloqueio parcial dos poros mais permeáveis com emulsão

Figura 2. 1 Tipos de emulsões em um sistema água e óleo (Miranda, 2010)....30 Figura 2. 2 Representação da molécula do surfactante.

www.sciencelearn.gov.nz 31

Figura 2. 3 Filme interfacial de surfactante. 32

Figura 2. 4 Formação do agregado micelar. 33

Figura 2. 5 Mecanismos que conduzem à instabilidade de uma emulsão.

(Adaptado de Alwadani, 2009).

Figura 2. 6 Frequência de aplicação de vários tipos de micromodelos, dos anos 50 até os anos 80

Figura 2. 7 Resumo das geometrias, materiais, processo de fabricação e campos de aplicação de micromodelos em estudos de escoamento bifásico. ...39

Figura 2. 8 Fluxo bifásico em um tubo capilar com comprimento e raio $r$ 44

Figura 2. 9 Curvas de pressão capilar na drenagem e na embebição (Guillén, 2011).

Figura 2. 10 Ângulo de contato de uma superfície hidrofílica/oleofóbica (à esquerda), de uma superfície hidrofóbica/óleofílica (direita) e de uma 
superfície com um grau de molhabilidade intermediaria (centro).

www.academia.edu.

Figura 2. 11 Efeito da A) concentração de Asfaltenos B) Água Salgada D)

Temperatura e de C) Tempo de envelhecimento; no ângulo de contato das lâminas de vidro. (Al Aulaqi et al., 2011).

Figura 2. 12 Micromodelo tratado com Decano (Vermelho=óleo, Amarelo= Água), Óleo (Marrom=óleo, Branco=Água), e Dimetildiclorossilano respectivamente. (Al Aulaqi et al., 2011).

Figura 2. 13 Efeito na mudança da molhabilidade do vidro com o tempo de envelhecimento em "Crude Oil". (Roosta et al.,2009) 52

Figura 2. 14 Distribuição de óleo residual: A) Micromodelo com superfície fortemente molhável à água B) Micromodelo com superfície fortemente molhável ao óleo (Tempo de envelhecimento: 15 dias) C) Micromodelo com superfície fortemente molhável ao óleo (depois da injeção de vapor). (Roosta et al.,2009).

Figura 3. 1 (a) Misturador mecânico rotativo Ultra-Turrax T-25 (b) Dispersor rotor-estator.

Figura 3. 2 (a) Esquema dos diferentes diâmetros de garganta encontrados no meio poroso, (b) Vista microscópica do meio poroso.

Figura 3. 3 Bomba de vácuo e filtros empregados nos processos de filtração das fases.

Figura 3. 4 Preparação da emulsão óleo em água $(\mathrm{O} / \mathrm{A})$ a injetar no meio poroso.

Figura 3. 5 Malvern MasterSizer (Modelo 2000). 62

Figura 3. 6 Distribuição de tamanho de gota da emulsão óleo em água. 63

Figura 3. 7 Estabilidade no tempo da emulsão de óleo em água injetada no micromodelo.

Figura 3. 8 Gota em equilíbrio entre a força gravitacional e a tensão superficial do líquido.

Figura 3. 9 Geometria de uma gota pendente. 65

Figura 3. 10 Tensiômetro TRACKER, baseado no método da gota pendente. ..67 Figura 3. 11 Tensão Interfacial Dinâmica do sistema fase contínua/óleo, $\mathrm{T}=23^{\circ} \mathrm{C}$

Figura 3. 12 Tensão Interfacial Dinâmica do sistema água destilada/óleo,

$\mathrm{T}=23^{\circ} \mathrm{C}$

Figura 3. 13 a) Tensiômetro TRACKER usado para medida da tensão 
interfacial, b) Goniômetro TRACKER usado para medida do ângulo de contato.

Figura 3. 14 Esquema ilustrativo do ângulo de contato formado entre a superfície de uma lâmina de vidro é uma gota de água. .................................70

Figura 3. 15 Limpeza requerida no micromodelo. ............................................... 71

Figura 3. 16 a) Micromodelo saturado com ar antes da saturação com solução diluída de DMDCS. b) Micromodelo saturado com solução diluída de DMDCS

Figura 3. 17 Ângulo de Contato Dinâmico da Interface ar/fase contínua, T = $23^{\circ} \mathrm{C}$

Figura 3. 18 Ângulo de Contato Dinâmico da Interface óleo/ fase contínua,

$\mathrm{T}=23^{\circ} \mathrm{C}$

Figura 3. 19 Bancada experimental montada para os experimentos realizados. 75

Figura 3. 20 Meio poroso utilizado na visualização do processo de deslocamento de óleo por injeção de emulsões. 76

Figura 3. 21 Dimensões externas do meio poroso de vidro..............................77

Figura 3. 22 Conexões para a montagem do meio poroso de vidro. .................. 77

Figura 3. 23 Meio poroso fixado às conexões............................................... 77

Figura 3. 24 Vista microscópica da estrutura interna do meio poroso. ................78

Figura 3. 25 Profundidade e largura dos canais no micromodelo....................... 78

Figura 3. 26 Tipos de canais formando a estrutura porosa do micromodelo. .....79

Figura 3. 27 Sistema de injeção de fluidos...................................................... 80

Figura 3. 28 Conexões entre a seringa e o micromodelo................................. 81

Figura 3. 29 Transdutor de pressão Validyne modelo DP - 15, placa de terminais $12871-1$ e placa de interface UPC2100. 81

Figura 3. 30 Instalação do ponto de tomada de pressão no micromodelo........... 82

Figura 3. 31 Microscópio Carl Zeiss Axiovert 40 MAT...................................... 83

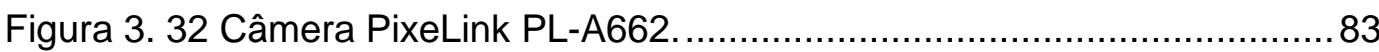

Figura 3. 33 Software para medição da distribuição de gota das emulsões. ......8 84

Figura 3. 34 Etapas do processo de limpeza com álcool etílico no micromodelo. 86

Figura 3. 35 Montagem das seringas nas bombas COLE PARMER. 88

Figura 3. 36 Circuito de Injeção das emulsões O/A. 89

Figura 4. 1 Dimensões da estrutura interna do micromodelo, utilizadas no cálculo geométrico do volume poroso 
Figura 4. 2 Comportamento da pressão média ponderada com a vazão. 93

Figura 4. 3 Esquema resumo dos experimentos realizados.............................94

Figura 4. 4 Área do micromodelo selecionada para sua posterior análise e visualização.

Figura 4. 4 Comportamento do fator de recuperação e pressão de injeção em função do número de volumes porosos injetados.

Figura 4. 6 Fotografias mostrando o processo de injeção de água e emulsão em uma seção do micromodelo. $\mathrm{Ca}=2 \times 10-5, \sigma=6.97 \mathrm{mNm}$.

Figura 4. 7 Fotografias mostrando o processo de injeção de água e emulsão em uma seção do micromodelo. $\mathrm{Ca}=2 \times 10-5, \sigma=6.97 \mathrm{mNm}$.

Figura 4. 8 Fotografias mostrando o processo de injeção de água e emulsão em uma seção do micromodelo. $\mathrm{Ca}=2 \times 10-5, \sigma=6.97 \mathrm{mNm}$.

Figura 4. 9 Fotografias mostrando o processo de injeção de água e emulsão em uma seção do micromodelo. $\mathrm{Ca}=2 \times 10-5, \sigma=6.97 \mathrm{mNm}$.

Figura 4. 10 Fotografias mostrando o processo de injeção de água e emulsão em uma seção do micromodelo. $\mathrm{Ca}=2 \times 10-5, \sigma=6.97 \mathrm{mNm}$ 102

Figura 4. 11 Fotografias mostrando o processo de injeção de água e emulsão em uma seção do micromodelo. Ca $=2 \times 10-5, \sigma=6.97 \mathrm{mNm}$ 103

Figura 4. 12 Fotografias mostrando o processo de injeção de água e emulsão em uma seção do micromodelo. $\mathrm{Ca}=2 \times 10-5, \sigma=6.97 \mathrm{mNm}$ 104

Figura 4. 13 Fotografias mostrando o processo de injeção de água e emulsão em uma seção do micromodelo. $\mathrm{Ca}=2 \times 10-5, \sigma=6.97 \mathrm{mNm}$ 105

Figura 4. 14 Fotografias mostrando o processo de injeção de água e emulsão em uma seção do micromodelo. $\mathrm{Ca}=2 \times 10-5, \sigma=6.97 \mathrm{mNm}$ 106

Figura 4. 15 Fotografias mostrando o processo de injeção de água e emulsão em uma seção do micromodelo. $\mathrm{Ca}=2 \times 10-5, \sigma=6.97 \mathrm{mNm}$ 107

Figura 4. 16 Regiões do micromodelo selecionadas para sua posterior análise de incertezas.

Figura 4. 17 Região três saturada com óleo à saturação irredutível de água ... 111

Figura 4. 18 Região três ao final do experimento de injeção de água.

Figura 4. 19 Região três ao final do experimento de injeção de um banco de emulsão

Figura 4. 20 Regiões do micromodelo selecionadas para sua posterior análise e visualização

Figura 4. 21 Comportamento do fator de recuperação de óleo mediante injeção de água e emulsão. 
Figura 4. 22 Comportamento da saturação de óleo residual mediante injeção de água e emulsão. 115

Figura 4. 23 Fotografias mostrando o processo de injeção de água (esquerda) e emulsão (direita). Região 2. $\mathrm{Ca}=2 \times 10-5, \sigma=6.97 \mathrm{mNm}$ 116

Figura 4. 24 Fotografias mostrando o processo de injeção de água (esquerda) e emulsão (direita). Região 3. Ca $=2 \times 10-5, \sigma=6.97 \mathrm{mNm}$

Figura 4. 25 Fotografias mostrando o processo de injeção de água (esquerda) e emulsão (direita). Região 2. $\mathrm{Ca}=1 \times 10-4, \sigma=6.97 \mathrm{mNm}$

Figura 4. 26 Fotografias mostrando o processo de injeção de água (esquerda) e emulsão (direita). Região 3. Ca $=1 \times 10-4, \sigma=6.97 \mathrm{mNm}$

Figura 4. 27 Comportamento do fator de recuperação de óleo mediante injeção de água e emulsão para as três regiões estudadas.

Figura 4. 28 Fotografias mostrando o processo de injeção de um VP de emulsão. $\mathrm{Ca}=1 \times 10-5, \sigma=6.97 \mathrm{mNm}$.

Figura 4. 29 Fotografias mostrando o processo de injeção de um VP de emulsão. $\mathrm{Ca}=1 \times 10-5, \sigma=6.97 \mathrm{mNm}$.

Figura 4. 30 Fotografias mostrando o processo de injeção de um VP de emulsão. $\mathrm{Ca}=1 \times 10-5, \sigma=6.97 \mathrm{mNm}$.

Figura 4. 31 Fotografias mostrando o processo de injeção de um VP de emulsão. $\mathrm{Ca}=1 \times 10-5, \sigma=6.97 \mathrm{mNm}$.

Figura 4. 32 Fotografias mostrando o processo de injeção de um VP de emulsão. $\mathrm{Ca}=1 \times 10-5, \sigma=6.97 \mathrm{mNm}$.

Figura 4. 33 Fotografias mostrando o processo de injeção de um VP de emulsão. $\mathrm{Ca}=1 \times 10-4, \sigma=6.97 \mathrm{mNm}$.

Figura 4. 34 Fotografias mostrando o processo de injeção de um VP de emulsão. $\mathrm{Ca}=1 \times 10-4, \sigma=6.97 \mathrm{mNm}$. 128

Figura 4. 35 Fotografias mostrando o processo de injeção de um VP de emulsão. $\mathrm{Ca}=1 \times 10-4, \sigma=6.97 \mathrm{mNm}$. 129

Figura 4. 36 Fotografias mostrando o processo de injeção de um VP de emulsão. $\mathrm{Ca}=1 \times 10-4, \sigma=6.97 \mathrm{mNm}$. 130

Figura 4. 37 Fotografias mostrando o processo de injeção de um VP de emulsão. $\mathrm{Ca}=1 \times 10-4, \sigma=6.97 \mathrm{mNm}$. 131

Figura 4. 38 Fotografias mostrando o processo de injeção de um VP de emulsão. $\mathrm{Ca}=1 \times 10-4, \sigma=6.97 \mathrm{mNm}$. 132

Figura 4. 39 Fotografias mostrando o processo de injeção de um VP de 
emulsão. $\mathrm{Ca}=1 \times 10-4, \sigma=6.97 \mathrm{mNm}$.

Figura 4. 40 Captura e acumulação de gotículas da emulsão O/A no meio

poroso.

Figura 4. 41 Ângulo de contato medido nas interfaces ar/fase contínua e óleo/ fase contínua em uma superificie de vidro.

Figura 4. 42 Fotografias mostrando o processo de injeção de água

(esquerda) e emulsão (direita). Micromodelo molhável à água. Ca $=2$ x 10-5, $\sigma=6.97 \mathrm{mNm}$.

Figura 4. 43 Fotografias mostrando o processo de injeção de água

(esquerda) e emulsão (direita). Micromodelo molhável ao óleo. Ca = 2 × 10-5, $\sigma=6.97 \mathrm{mNm}$. 


\section{Lista de Tabelas.}

Tabela 2. 1 Métodos de medição de molhabilidade (Barclay, 2000). .47

Tabela 2. 2 Restauração da molhabilidade com a exposição ao vapor. .53

Tabela 3. 1 Propriedades do Surfactante STEOL CS-330.

http://www.stepan.com/pdfs/Bulletins/STEOLCS330.pdf 55

Tabela 3. 2 Propriedades do óleo mineral AGECOM 500PS a temperatura de $25^{\circ} \mathrm{C}$.http://www.agecom.com.br/produtos/quimica/oleos-minerais-basicos...... 55 Tabela 3. 3 Propriedades físicas e químicas do Diclorodimetilsilano, DMDCS. http://www.sigmaaldrich.com/MSDS/MSDS/DMDCS .57

Tabela 3. 4 Valor médio e desvio padrão das medições dinâmicas. .................. 74

Tabela 3. 5 Características gerais do meio poroso de vidro............................. 79

Tabela 4. 1 Volume poroso e porosidade dos diferentes testes. .92

Tabela 4. 2 Vazões e diferenciais de pressão usados para o cálculo da permeabilidade absoluta do micromodelo. .92

Tabela 4. 3 Saturações e fatores de recuperações obtidos para o cálculo das incertezas nos experimentos. 109

Tabela 4. 4 Variação das saturações em diferentes regiões do micromodelo. .110 Tabela 4. 5 Comparação das saturações e fatores de recuperação para os dois casos de molhabilidade estudada. 